\title{
PARTICIPAÇÃO DOS HOMENS EM ATIVIDADES DE RASTREAMENTO DO CÂNCER BUCAL: UM RELATO DE EXPERIÊNCIA
}

\author{
José Nunes Carneiro Neto \\ Universidade Estadual de Feira de Santana \\ jnunes.neto@yahoo.com.br \\ Michelle Miranda Lopes Falcão \\ Universidade Estadual de Feira de Santana \\ michellefalcao@gmail.com
}

\author{
Valéria Souza Freitas \\ Universidade Estadual de Feira de Santana \\ valeria.souza.freitas@gmail.com
}

Resumo

O câncer bucal é um problema de saúde pública. O rastreamento pode revelar-se medida importante de controle da doença, visto que a maioria dos casos é detectada em fase avançada. A morbidade é maior em homens acima de 40 anos, baixa renda, etilistas e tabagistas. Objetivo Relatar a experiência da participação masculina nas atividades extensionistas relacionadas ao rastreamento do câncer bucal desenvolvidas através do Programa de Prevenção e Controle do Câncer de Boca no Município de Feira de Santana, Bahia. Método Durante um ano realizou-se 11 atividades de rastreamento de lesões bucais voltadas ao público masculino em Feira de Santana. Resultados Participaram das atividades 593 pessoas, sendo $204(34,4 \%)$ homens. Encaminhou-se para atendimento ambulatorial ou tratamento em centros de maior complexidade 8,7\% (51) dos participantes. Considerações finais A baixa participação masculina nessas atividades reforça a necessidade de continuar com ações educativas para reduzir a prevalência da doença nessa população.

Palavras-chave: Programas de Rastreamento. Educação em saúde. Saúde do homem.

\section{PARTICIPATION OF MEN IN ORAL CANCER SCREENING FOR ACTIVITIES:}

\section{AN EXPERIENCE REPORT}

\begin{abstract}
Oral cancer is a public health problem. The screening may be found to be a measure for controlling this disease, since most cases are detected at advanced stage. The most morbidity is men aged over 40 years, with low income, alcohol abusers and smokers. Objective To report the experience of male participation of extension activities relating to oral cancer screening developed through the Oral Cancer Prevention and Control Program of the municipality of Feira de Santana, Bahia. Method Over a one-year, 11 oral lesion screening activities were conducted, targeted towards men in Feira de Santana. Results There were 593 participants in the activities, of whom 204 (34.4\%) were male and 8.7\% (51) were referred for outpatient care or for treatment at high-complexity centers. Conclusion The low participation of the male in these activities reinforces the need to continue with educational activities of reducing the prevalence of disease in this population. Keywords: Mass Screening. Health Education. Men's health.
\end{abstract}

\section{PARTICIPACIÓN DE LOS HOMBRES EN TAMIZAJE PARA CÁNCER ORAL: UN RELATO DE EXPERIENCIA}

Resumen

El cáncer oral constituye un problema de salud pública. Ocurre con mayor frecuencia en hombres mayores de 40 años, bajos ingresos, consumidores de alcohol y fumadores. El tamizaje es una posible medida para el control de esa enfermedad. Objetivo Reportar la participación de los hombres en actividades de extensión relativas a la detección del cáncer oral, desarrollado a través del Programa de Prevención y Control del Cáncer de boca en la ciudad de Feira de Santana, Bahía. Método Durante un año fueron realizados 11 tamizajes para la identificación de lesiones orales en hombres de Feira de Santana. Resultados De los 593 participantes, 204 (34,4\%) eran hombres y 8,7\% (51) fueron remitidos para atención ambulatoria o para tratamiento en centros de alta complejidad. Conclusión La baja participación masculina en estas actividades refuerza la necesidad de la continuación del tamizaje, visando contribuir em la reducción de la prevalencia del cáncer oral.

Descriptores: Tamizaje Masivo. Educación en Salud. Salud del Hombre. 


\section{INTRODUÇÃO}

O câncer bucal é um importante problema de saúde pública no Brasil e no mundo. A maioria dos fatores de risco está relacionada a hábitos de vida como a presença do tabagismo, do etilismo e da ausência do uso de proteção durante a exposição solar (SCULLY, FELIX, 2006; OLIVEIRA et. al., 2010), além disso, é mais frequente em homens acima dos 40 anos. Trata-se de uma doença mutilante que pode levar a morte, cujo tratamento revela-se de alto custo econômico e social (KOWALSKI et. al., 1991). De acordo com as últimas estimativas do Instituto Nacional do Câncer, ocupa o $5^{\circ}$ lugar entre as dez neoplasias malignas que mais acometem o sexo masculino, representando 3,7\% dos casos novos (BRASIL, 2015). Há alguns anos ocupou o $8^{\circ}$ lugar.

A explicação para o aumento dessa morbidade pode estar associada a diversos fatores, entre eles, o perfil masculino em relação à percepção do processo saúde doença e medidas de prevenção e controle. De acordo com Figueiredo (2005), o homem tende a desvalorizar o autocuidado, preocupando-se pouco com a saúde, além disso, considera a presença de doenças como sinal de fragilidade, o que muitas vezes o paralisa, impedindo-o de buscar o auxílio médicoodontológico precocemente (BRASIL, 2008; CORDEIRO et. al., 2011). Como consequência, adentra ao sistema de saúde, através do nível secundário de atenção, o que incide em tratamento mais oneroso e prognóstico menos favorável para a maioria das doenças, quando comparado ao atendimento na atenção básica (BRASIL, 2008).

O rastreamento, como um procedimento capaz de auxiliar na redução dos indicadores de morbimortalidade do câncer bucal, surge como uma opção ao diagnóstico precoce dessa doença, uma vez que, compreende uma manobra de busca ativa dos casos assintomáticos de uma população que auxilia na identificação de fatores de risco, de lesões com potencial de malignização e de câncer bucal, com posterior encaminhamento dos indivíduos com lesões suspeitas para investigação diagnóstica em um serviço de saúde (SCHEUFEN et. al., 2011; BRASIL, 2010; RETHMAN et. al., 2010; WORLD HEALTH ORGANIZATION, 2002).

Nesse sentido, o estímulo à participação masculina nesse tipo de atividade poderá reverter o atual perfil epidemiológico da doença, ao possibilitar o exame clínico rápido desse grupo específico, a sua sensibilização quanto aos fatores de risco para o câncer bucal (BROWN et. al., 1988) e à realização do autoexame da boca, haja vista o comportamento masculino frente ao cuidado em saúde (FIGUEIREDO, 2005). O objetivo deste trabalho é relatar a experiência da participação masculina nas atividades extensionistas relacionadas ao rastreamento do câncer bucal desenvolvidas pelo Núcleo de Câncer Oral (NUCAO), da Universidade Estadual de Feira de 
Santana (UEFS), através do Programa de Prevenção e Controle do Câncer de Boca no Município de Feira de Santana-BA.

\section{MATERIAIS E MÉTODOS}

Trata-se de um relato de atividade extensionista, relacionada ao Programa de Prevenção e Controle do Câncer de Boca no Município de Feira de Santana-BA. Esse Programa iniciou suas atividades em 1998, desde então, tem auxiliado na redução dos indicadores de morbimortalidade do câncer de boca da região, através da realização de atividades educativas, de rastreamento de lesões bucais e da capacitação de diversos profissionais de saúde sobre a temática.

Ao observar a baixa participação masculina nas atividades educativas e de rastreamento do câncer de boca, lesões e desordens com potencial de malignização, decidiu-se realizar, durante um ano, algumas oficinas de saúde voltadas a esse público, com o intuito de sensibilizá-los à adoção de medidas preventivas como a redução/abandono dos hábitos tabagistas, etilistas, o uso de proteção solar e a realização periódica do autoexame de boca.

Para isso, inicialmente, houve o treinamento da equipe sobre rastreamento e abordagem da temática com o público masculino, seguida da exposição do projeto e agendamento das oficinas de saúde em espaços que possibilitasse a maior participação dos homens. Dessa forma, foram realizadas 11 atividades educativas e de rastreamento destinadas aos homens do município de Feira de Santana - Bahia. Os responsáveis pelos espaços responsabilizaram-se pela entrega prévia dos convites para as ações educativas destinadas à população local, principalmente, de indivíduos do sexo masculino, de modo a viabilizar a participação dos mesmos nas atividades (Quadro 1).

\begin{tabular}{|l|r|r|}
\hline \multicolumn{1}{|c|}{ LOCAL DA ATIVIDADE } & \multicolumn{2}{c|}{ TOTAL DE PARTICIPANTES } \\
\cline { 2 - 3 } & $\mathbf{n}$ & $\mathbf{0}$ \\
\hline II Feira de Saúde da Universidade Aberta da Terceira Idade & 84 & 14,0 \\
Centro Paroquial da Igreja Católica de Anguera & 38 & 6,5 \\
Escola Estadual Juiz Jorge Farias Góes & 66 & 11,0 \\
Escola Municipal Rosa Maria Esperidião Leite & 82 & 13,8 \\
Associação Presbiteriana de Ação Social & 84 & 14,0 \\
Unidade de Saúde da Família do Bairro Gabriela II & 36 & 6,2 \\
Escola Estadual Juiz Jorge Farias Góes & 27 & 4,6 \\
Unidade de Saúde da Família do Distrito de Tiquaruçu & 26 & 4,5 \\
Polo da Cidadania & 66 & 11,0 \\
\end{tabular}



RELATO DE EXPERIÊNCIA

\begin{tabular}{|c|c|r|} 
Canteiro de obras da construção civil & 24 & 4,2 \\
I Feira de Saúde do Centro de Abastecimento & 60 & 10,2 \\
\hline TOTAL & 593 & 100 \\
\hline
\end{tabular}

Quadro 1 - Distribuição do local e público participante das atividades educativas e de rastreamento de câncer bucal em Feira de Santana entre 2011 e 2012.

Fonte: Elaborado pelos autores, 2015.

As atividades foram realizadas no período de dezembro de 2011 a dezembro de 2012 pelos estudantes e pesquisadores vinculados ao NUCAO/UEFS. Todo o material didáticopedagógico utilizado foi confeccionado pelos bolsistas sob a supervisão dos orientadores. Cada atividade teve a duração média de 120 minutos, sendo 40 minutos destinados à educação em saúde sobre o câncer bucal e 80 minutos ao rastreamento de lesões e condições bucais com potencial de malignização e câncer bucal. As atividades foram realizadas no espaço físico alocado pelas instituições parceiras.

A educação em saúde sobre o câncer bucal contemplou: a) exposição oral de álbum seriado; b) distribuição de folders educativos; e c) dinâmica de pergunta e resposta acerca dos fatores de risco e proteção para o câncer bucal com uso de cartões vermelhos e verdes para discordância e concordância, respectivamente. A linguagem educativa adequou-se ao nível sociocultural do público-alvo. Os instrumentos para avaliação do nível de conhecimento dos participantes, principalmente os indivíduos do sexo masculino, incluíram exposição de idéias e pergunta sobre o câncer bucal antes e após a abordagem do tema.

Para a realização da atividade educativa, propriamente dita, no primeiro momento, com intuito de motivar os participantes e auxiliar na aproximação dos mesmos com o tema, além de melhorar a interação com a equipe, foi realizada uma dinâmica dos fatores de risco e proteção para o câncer de boca com a apresentação de imagens ilustrativas sobre os fatores de risco e proteção do câncer de boca. À medida que se mostrava a figura, os participantes informavam se tratava ou não de fatores/condições de risco para o câncer bucal.

Com essa atividade, foi possível levantar o nível de informação dos participantes sobre o tema e direcionar a apresentação expositiva do álbum seriado de acordo com a realidade. Assim, os temas abordados contemplaram a definição do câncer bucal, fatores etiológicos, atitudes preventivas, autoexame bucal, fatores de risco do câncer de boca e comportamento masculino em relação à saúde.

Aproveitou-se o momento das atividades para orientar sobre higiene bucal com uso de macromodelo de boca, escova, fio dental e materiais alternativos de higiene bucal. Após a explanação dos temas, houve sorteio de kits de higiene bucal através do número da lista de 
presença. Posteriormente, foi realizado o exame físico intraoral de todos os indivíduos que consentiram participar do mesmo.

Para o rastreamento foi utilizado cadeira odontológica simplificada, equipamentos de proteção individual - EPI, gaze e espátula de madeira, iluminação natural dos espaços físicos das atividades. Os participantes identificados com suspeitas de lesões/desordens bucais com potencial de malignização e câncer de boca foram encaminhados para o Centro de Referência de Lesões Bucais/UEFS para melhor avaliação clínica e tratamento ou encaminhamento dos casos confirmados de câncer para atendimento nas Unidades de Assistência de Alta Complexidade em Oncologia - UNACON.

\section{RESULTADOS E ANÁLISES}

Participaram das 11 atividades educativas e de rastreamento 593 pessoas, sendo apenas $204(34,4 \%)$ do sexo masculino, mesmo tendo sido explicado no momento do agendamento das atividades a necessidade da presença do sexo masculino, por esse compor o grupo de risco para câncer de boca (SCULLY; FELIX, 2006; BRASIL, 2015) (FIGURA 1). Esse fato retrata a baixa participação dos homens em atividades não laborais e aponta a necessidade da realização de ações que incentivem a participação desse grupo, haja vista os indicadores de morbimortalidade do câncer bucal (BRASIL, 2015) e desvalorização do autocuidado com a saúde (FIGUEIREDO, 2005).

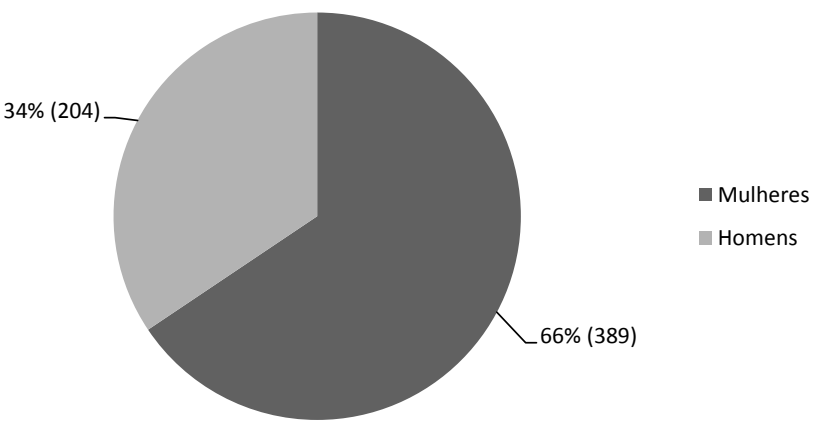

Figura 1 - Distribuição dos participantes das atividades de educação em saúde e rastreamento de leões bucais, segundo sexo, Feira de Santana, Bahia, no período de 2011 a 2012. Fonte: Elaborada pelos autores, 2015. 
Percebeu-se que a dinâmica inicial proposta com o intuito de acolher e reduzir a timidez dos participantes obteve um resultado positivo, pois houve ampla participação do público no que tange a explanação de ideias, fato que pode auxiliar na redução dos indicadores de morbimortalidade do câncer de boca através da sensibilização sobre os fatores de risco e autoexame bucal (SASCO, SECRETAN, STRAIF, 2004). A abordagem da higiene bucal teve o propósito de trabalhar a integralidade das ações em saúde e orientar sobre as estruturas que compõem a cavidade bucal, compondo uma das metas na política de saúde do homem (BRASIL, 2008).

Foram encaminhados para atendimento ambulatorial ou para tratamento no UNACON $8,7 \%$ (51) do total de indivíduos rastreados, sendo desses 2,6\% (15) do sexo masculino. Eles apresentaram, no momento do exame, lesões brancas, vermelhas e negras, além disso, referiram baixa renda familiar, presença de hábitos etilistas e de consumo de tabaco, situação que corrobora com os achados da literatura sobre os fatores/condições de risco para o câncer de boca (CORDEIRO et. al., 2011; BRASIL, 2008; FERNANDES et. al., 2008; VOLKWEIS et. al., 2010; SILVEIRA et. al., 2009; RODRIGUES et. al., 2000; MARTINS et. al., 2008; SILVA et. al., 2007; HOSNI et. al., 2009; OLIVEIRA et. al., 2010).

Diante dos resultados, observa-se que mesmo com o planejamento das atividades de rastreamento de modo a possibilitar a participação do grupo de risco para o câncer bucal, isto é, os homens maiores de 40 anos, etilistas e tabagistas, não se obteve êxito na maior frequência de homens nessas atividades. Essa situação leva a refletir sobre a necessidade da implementação sistemática e contínua de ações que auxiliem na mudança do comportamento masculino em relação ao cuidado em saúde, de modo a desconstruir padrões culturais instituídos ao longo das gerações, reconstruindo-os de forma salutar.

\section{CONSIDERAÇÕES FINAIS}

A baixa participação de indivíduos do sexo masculino nas ações educativas, associada aos indicadores de morbimortalidade do câncer de boca reforça a necessidade de continuidade das atividades de educação em saúde e de rastreamento para esse grupo, com o intuito de reduzir a prevalência do câncer bucal e, consequentemente, o número de mortes precoces e mutilações desnecessárias. 


\section{REFERÊNCIAS}

BRASIL. INSTITUTO NACIONAL DO CÂNCER. 2015 [online]. Estimativas 2014. Disponível em: <http://www.inca.gov.br/estimativa/2014/tabelaestados.asp?UF=BR>. Acesso em: 30 mar. 2015.

BRASIL. Ministério da Saúde. Secretaria de Atenção a Saúde. Departamento de Ações Pragmáticas Estratégicas. Política Nacional de Atenção Integral à Saúde do Homem (Princípios e Diretrizes), Brasília, 2008.

BRASIL. Ministério da Saúde. Secretaria de Atenção à Saúde. Departamento de Atenção Básica. Rastreamento [Série A. Normas e Manuais Técnicos, Cadernos de Atenção Primária, n. 29]. Brasília, 2010.

BROWN, L. M. et. al. Ambient factors and high risk of esophageal cancer between men in coast South Carolina. Installation National of the cancer Journal, v. 80, p. 1620-1625, 1988.

CORDEIRO, S. B. M. et. al. Características sociodemográficas e condições de saúde da população urbana de Feira de Santana, Bahia: análise de diferenciais de gênero. Revista Baiana de Saúde Pública, v. 35, supl.1, p. 9-27, 2011.

FERNANDES, J. P. et. al. Prevalência de lesões cancerizáveis bucais em indivíduos portadores de alcoolismo. Revista Brasileira de Cancerologia, v. 54, n. 3, p. 239-244, 2008.

FIGUEIREDO, W. Assistência à saúde dos homens: um desafio para os serviços de atenção primária. Revista Ciência e Saúde Coletiva, v. 10, n. 1, p. 105-109, 2005.

HENRIQUE, P. R. et. al. Prevalência de alterações da mucosa bucal em indivíduos adultos da população de Uberaba, Minas Gerais. Revista Gaúcha de Odontologia, Porto Alegre, v. 57, n.3, p. 261-267, 2009.

HOSNI, E. S. et. al. Eritroplasia e leucoeritroplasia oral: análise retrospectiva de 13 casos. Brazilian Journal of Otorhinolaryngology, São Paulo, v. 75, n. 2, p. 295-299, 2009.

KOWALSKI, L. P. et. al. Modelo de programa de prevenção e detecção precoce do câncer bucal. Saúde em Debate, v. 32, n. 3, p. 66-71, 1991.

MARTINS, R. B. et. al. Lesões cancerizáveis na cavidade bucal. Revista do Instituto de Ciência e Saúde, São Paulo, v. 26, n. 4, p. 467-476, 2008.

OLIVEIRA, L. G. S. et. al. Prevalência de lesões bucais cancerosas e cancerizáveis em pacientes ambulatoriais atendidos no FBHC de 2006 a 2007. Odontologia Clínica e Científica, Recife, v. 9, n. 2, p. 145-150, 2010.

RETHMAN, M. P. et. al. Evidence-based clinical recommendations regarding screening for oral squamous cell carcinomas. Journal American Dental Association, v. 141, n. 5, p. 509-20, 2010.

RODRIGUES, T. L. C. et. al. Leucoplasias bucais: relação clínico-histopatológica. Pesquisa Odontológica Brasileira, v. 14, n. 4, p. 357-361, 2000. 
SASCO, A. J.; SECRETAN, M. B.; STRAIF, K. Tobacco smoking and cancer: a brief review of recent epidemiological evidence. Lung Cancer, v. 45, suppl. 2, p. 03-09, 2004.

SCHEUFEN, R. C. et. al. Prevenção e detecção precoce do câncer de boca: sreening em populações de risco. Pesquisa Brasileira de Odontopediatria e Clínica Integrada, v. 11, n. 2, p. 245-249, 2011.

SCULLY, C.; FELIX, D. H. Oral Medicine. Update for the dental practitioner. Oral cancer. British Dental Journal, v. 200, n. 1, p. 13-17, 2006.

SILVA, I. C. O. et. al. Leucoplasia: uma revisão de literatura. Revista Gaúcha de Odontologia, Porto Alegre, v. 55, n. 3, p. 287-289, 2007.

SILVEIRA, E. J. D. et. al. Lesões orais com potencial de malignização: análise clínica e morfológica de 205 casos. Jornal Brasileiro de Patologia Médica e Laboratorial, v. 45, n. 3, p. 233-238, 2009.

VOLKWEIS, M. R. et. al. Estudo retrospectivo sobre as lesões bucais na população atendida em um Centro de Especialidades Odontológicas. Revista Gaúcha de Odontologia, Porto Alegre, v. 58, n.1, p. 21-25, 2010.

WORLD HEALTH ORGANIZATION. National Cancer Control Programmers: policies and managerial guidelines. 2 ed. WHO: Geneva, 2002. Disponível em <http://whqlibdoc.who.int/hq/2002/9241545577.pdf?ua=1>. Acesso em: 24 jul. 2015. 